\title{
The Role of Tracheal Smooth Muscle Contraction on Neonatal Tracheal Mechanics
}

\author{
RANDY J. KOSLO, VINOD K. BHUTANI, AND THOMAS H. SHAFFER \\ Department of Physiology, Temple University School of Medicine, and Section on Newborn Pediatrics, \\ Pennsylvania Hospital, University of Pennsylvania, Philadelphia, Pennsylvania 19140
}

\begin{abstract}
The ability of tracheal smooth muscle tone to modulate the mechanical properties of neonatal airways was evaluated in six newborn lambs. Tracheal pressurevolume relationships, isovolumic compliance, hysteresis, and the relaxation time constant of the smooth muscle were evaluated as a function of incremental cholinergic stimulation. Tracheal active tensions were also determined at the graded levels of cholinergic stimulation. Data show that a maximal cholinergic stimulation resulted in a mean developed active tension value of $14.3 \pm 2.13 \mathrm{SEM} \times 10^{3}$ dynes $/ \mathrm{cm}$. The resultant $55 \%$ decrease in tracheal compliance was linearly correlated to the increase in active tension $(r=0.90, p<0.01)$. Cholinergic stimulation also resulted in a $18.4 \%$ decrease $(p<0.02)$ in the relaxation time constant of the smooth muscle, and in a $82 \%$ increase ( $p$ $<0.01$ ) in tracheal hysteresis. This study demonstrates that both elastic and viscoelastic properties of the neonatal lamb trachea are effected by tracheal smooth muscle contraction. Since neonatal airways are more compliant than adult airways, cholinergic stimulation could conceivably enhance airway rigidity and may provide a protective mechanism against pressure-induced deformation. (Pediatr Res 20: 1216-1220, 1986)
\end{abstract}

Abbreviation

P-V, pressure-volume

Tracheal smooth muscle tone plays an important and significant role in the conductance and the dimensions of the adult tracheobronchial tree (1). Coburn and Palombini (2) have demonstrated that graded increases in the active tension of the trachealis muscle results in a proportional decrease in the adult canine tracheal compliance. Other effects of smooth muscle tension on adult tracheal mechanics have been documented with respect to its viscoelastic properties (3), regulation (4), and structure-function (5). It is well accepted that neonatal airways are compliant structures $(6,7)$. Although these in vitro studies have provided valuable information concerning the passive properties of the airways, the effect of smooth muscle tone on the functional behavior of neonatal airways is not known. The relationship between smooth muscle contraction and mechanical function of the immature trachea has not been evaluated, hence it is unclear whether airway rigidity and function can be controlled at early stages of development.

The purpose of this study was to evaluate the role of tracheal smooth muscle tone on the tracheal mechanics of term newborn

Received December 2, 1985; accepted June 30, 1986.

Send correspondence and reprint requests to Randy J. Koslo, Ph.D., c/o Thomas H. Shaffer, Ph.D., Temple University School of Medicine, Department of Physiology, 3420 North Broad Street. Philadelphia, PA 19140.

Supported in part by NIH Grant HL 32031 . lambs. The in vivo contraction responses of the trachea to cholinergic stimulation was utilized to alter smooth muscle tone and its effect on tracheal elastic and viscoelastic behavior were determined. The relationship between tracheal compliance, hysteresis, and relaxation time constant of the smooth muscle were evaluated as a function of the change in tracheal smooth muscle tension.

\section{METHODS}

Animal preparation. Six full-term newborn lambs, 1 to 3 days postpartum, were anesthetized $(30 \mathrm{mg} / \mathrm{kg}$ intraperitoneal pentabarbital) and secured in the supine position. The ventral surface of the neck was dissected to reveal the upper trachea, and the exposed tracheal area proximal to the thoracic cage was transected and cannulated to allow spontaneous ventilation. Vascular integrity was maintained and subsequently verified by cholinergic stimulation. The left jugular vein was cannulated, and electrocardiographic leads were attached to the lamb.

Proximal tracheal segments of $2.8 \mathrm{~cm}$ in length were measured and isolated. A plug was inserted in the most caudal end of the bypassed segment (Fig. 1). The lambs were then intubated with an endotracheal tube secured in place with ligatures at the cricoid cartilage. A rigid frame was affixed externally to the bypassed segments to maintain constant length during the experimental procedures.

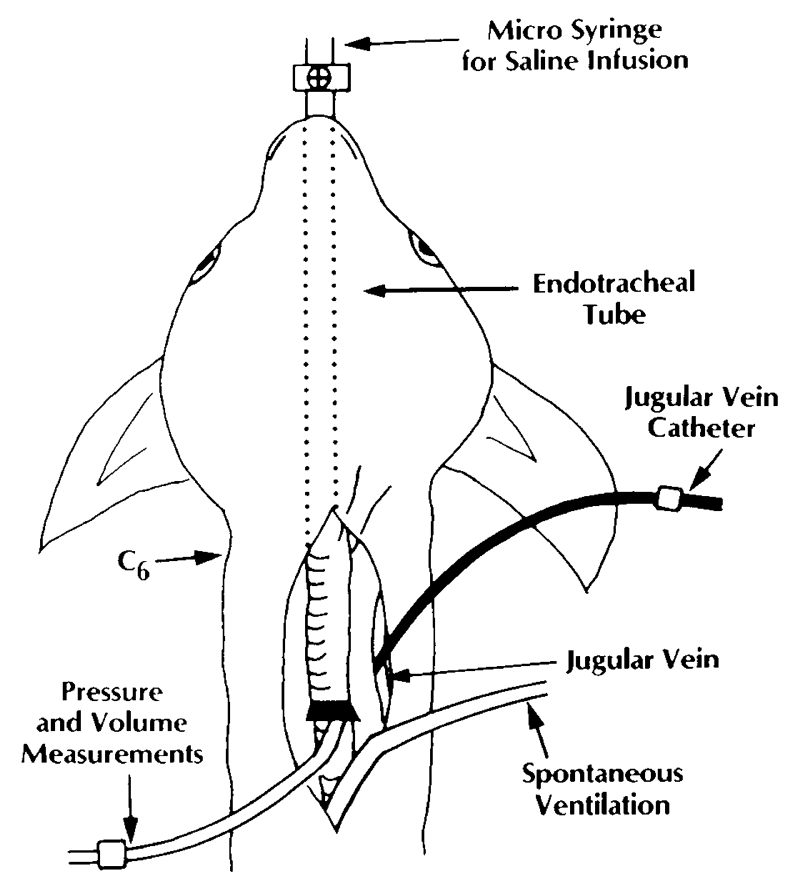

Fig. 1. Schematic of the experimental preparation. 
The distal end of the bypassed tracheal segment was cannulated, and connected to a micro-pipet and a pressure transducer (Statham P23db). The transducer signal was monitored and recorded on the Grass polygraph. A two-way valve was used to open and close the tubing circuit connected to the micro-pipet. An injection system (Hamilton micro-syringe and injector) was attached to the proximal end of the endotracheal tube and utilized for infusion and withdrawal. The bypassed tracheal segment and the entire circuit was filled with normal saline after being flushed of any mucus or secretions. Care was taken to minimize any net hydrostatic effect on the pressure transducer.

Pressure-volume relationships. The bypassed tracheal segment was initially opened to ambient pressure under control conditions. This allowed equilibration of pressures such that the transmural pressure across the wall of the trachea was at a baseline of zero. The system was then closed to atmosphere. Normal saline was injected into the system until an intratracheal pressure of 80 $\mathrm{cm} \mathrm{H}_{2} \mathrm{O}$ water was produced. The saline volume required to produce this pressure change was measured and then divided into four equal aliquots. Utilizing the Hamilton injection system, these four equivalent volumes were first introduced into the closed tracheal system at 15 -s intervals, and then removed over the same time period. Pressure changes concurrent to the stepvolume introduction and withdrawal were monitored. Similarly, the volume required for complete tracheal segmental collapse was also measured, and divided into four aliqouts. These four equivalent volumes were withdrawn and reintroduced to determine pressure-volume data at negative transmural pressures. Figure 2 illustrates the resulting pressure changes due to the stepvolumes. When a step-volume was added to the resting isolated tracheal segment, there was a concurrent rise in pressure. This pressure response typically peaked and then decreased to a stable plateau.

Values of the steady state pressure concomitant to the change in volume were used to construct $\mathrm{P}-\mathrm{V}$ relationships. The quasistatic P-V paradigm was utilized to determine tracheal compliance, hysteresis, and the relaxation time constant of the smooth muscle.

Experimental protocol. After the control pressure-volume measurements were obtained, the closed tracheal segment was again opened to ambient pressure. The micropipet was then

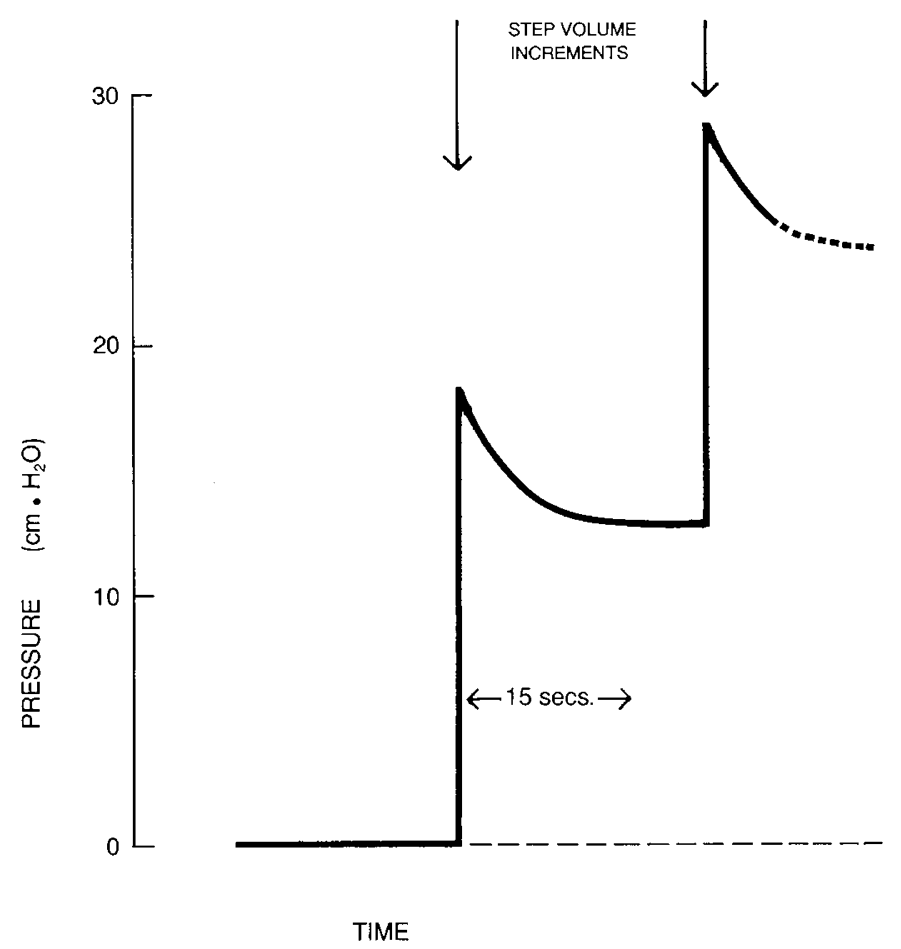

Fig. 2. Pressure response due to $(0.25 \mathrm{ml})$ step-volume increments. placed in series with the saline-filled tracheal segment. Carbamyl methylcholine (Bethanecol), a cholinergic agonist, was selected as the tracheal smooth muscle stimulant. Incremental dosages of $0.04 \mathrm{mg} / \mathrm{kg}$, to a maximum cumulative dosage of $0.16 \mathrm{mg} / \mathrm{kg}$, were administered through the jugular vein catheter. The ensuing changes in volume due to the tracheal smooth muscle contraction were measured in the micropipet. After each dosage of Bethanecol, the tracheal segment volume was reestablished and the tracheal system was closed to the atmosphere. The pressurevolume relationship of the bypassed segment was determined for each new level of smooth muscle active tension. Thus, the pressure-volume relationships of the trachea were determined in the control state and at each of the four levels of drug administration.

Graded smooth muscle contractile responses were observed in a series of preliminary experiments and associated with the administration of the cumulative dosages of Bethanecol. Similar dose response relationships have been previously observed with the tracheal smooth muscle of adults $(2,8)$.

The tracheal diameter was measured in situ after the experimental procedures. Utilizing this measurement and the known segment length, the final volume of the tracheal segment was determined. The initial control volume was then calculated by summing the final determined volume and the amount of the volumes expelled after Bethanecol administration.

Data reduction. The pressure-volume data were plotted using standard methods, and all data were calculated at isovolumic (control volume) values. Tracheal compliance was determined as the change in pressure between 95 and $105 \%$ of the control volume along the deflation curve. This technique was applied to both the control and the experimental conditions.

As described by Coburn and Palombini (2), developed active tension was calculated for both the inflation and deflation curves on the positive pressure limb of the P-V relationship. Since the trachea was assumed to be cylindrical, the law of LaPlace was implemented in the determination of active tension. The law of LaPlace states that tension $(\mathrm{T})$ equals pressure $(\mathrm{P})$ times radius (r) or:

$$
\mathrm{T}=\mathrm{P} \times \mathrm{r}
$$

The developed active tension was calculated at the isovolumic points and reported as a relative increase in tension. In our data analysis, the pressure difference between the control and stimulated trachea at isovolumic points was attributed to the contraction of the tracheal smooth muscle.

Based on regresssion analysis, the exponential pressure decay following an incremental step volume to the tracheal segment (Fig. 2) was described as:

$$
\mathrm{P}=\mathrm{P}_{\mathrm{ss}}+\left(\mathrm{P}_{0}-\mathrm{P}_{\mathrm{ss}}\right) \mathrm{e}^{-\mathrm{t}} / \tau_{\mathrm{R}},
$$

where $P=$ pressure at any given time $(t), P_{o}$ is the pressure at time zero (experimentally extrapolated), $P_{s s}=$ pressure at steady state conditions and $\tau_{\mathrm{R}}$ is the smooth muscle relaxation time constant.

Hysteresis $(\mathrm{g} \cdot \mathrm{cm})$ of the tracheal segment was determined to be the area within the positive pressure-volume loop of the plotted pressure-volume data.

Two types of statistical analyses were used in this study. Control and experimental data were compared for significance using the analysis of variance. To further study the interrelationships between various experimental parameters, linear regression analysis were performed.

\section{RESULTS}

The mean control tracheal volume for the newborn lambs was $3.42 \pm 0.5 \mathrm{SEM}$ ml. After each dose of Bethanechol $(0.04 \mathrm{mg} /$ $\mathrm{kg}$ ) there was a transient expulsion of saline followed by a steady state volume. At maximal Bethanechol stimulation $(0.16 \mathrm{mg} /$ $\mathrm{kg})$ the tracheal volume decreased $11.6 \%(p<0.01)$. Maximal increases in the developed active tension were determined for 
isovolumic pressure changes and are presented for individual segments (Table 1). The mean active tension value determined for the deflation positive pressure curve following maximal $\mathrm{Be}$ thanechol stimulation $(0.16 \mathrm{mg} / \mathrm{kg})$ was $14.3 \pm 2.13 \mathrm{SEM} \times 10^{3}$ dynes/cm.

The typical pressure-volume data for a single experiment are plotted and illustrated in Figure 3. These data exhibit a characteristic sigmoid shape with varying hysteresis during inflation and deflation for each condition studied. Resting volumes at zero transmural pressure are shown to decrease with increasing active tension, causing the curves to shift downward. Although in several cases the inflation and deflation limbs did not return to zero transmural pressure, this was not a typical response, and therefore has not been illustrated in Figure 3.

Under isovolumic conditions tracheal compliances exhibited

Table 1. Effect of maximal tracheal smooth muscle contraction* on tracheal compliance and developed active tension

\begin{tabular}{|c|c|c|c|}
\hline \multirow[b]{2}{*}{$\begin{array}{l}\text { LAMB } \\
\text { NUMBER }\end{array}$} & Control & \multicolumn{2}{|c|}{ Experimental } \\
\hline & $\begin{array}{c}\text { Compliance } \\
\left(\times 10^{-2} \mathrm{ml} / \mathrm{cm} /\right. \\
\left.\mathrm{H}_{2} \mathrm{O}\right)\end{array}$ & $\begin{array}{c}\text { Active } \\
\text { tension } \\
\left(\times 10^{3} \text { dynes/ }\right. \\
\mathrm{cm})\end{array}$ & $\begin{array}{c}\text { Compliance } \\
\left(\times 10^{-2} \mathrm{ml} / \mathrm{cm} \mathrm{H}_{2} \mathrm{O}\right)\end{array}$ \\
\hline I & 2.90 & 2.66 & 2.20 \\
\hline II & 4.30 & 11.93 & 1.10 \\
\hline III & 2.20 & 19.93 & 0.80 \\
\hline IV & 1.20 & 2.84 & 0.50 \\
\hline V & 2.00 & 14.88 & 0.90 \\
\hline VI & 3.40 & 34.30 & 0.90 \\
\hline Mean & 2.70 & 14.30 & 1.07 \\
\hline \pm SEM & \pm 2.13 & \pm 2.13 & \pm 0.20 \\
\hline
\end{tabular}

* Maximal contraction determined at a dosage $0.16 \mathrm{mg} / \mathrm{kg}$ Bethanecol decreases with increasing active tension, and are presented in Table 1. The mean control compliance was $2.70 \pm 0.40 \times 10^{-2}$ $\mathrm{ml} / \mathrm{cm} \mathrm{H}_{2} \mathrm{O}$ and decreased $55 \%(p<0.02)$ to a mean of $1.07 \pm$ $0.20 \times 10^{-2} \mathrm{ml} / \mathrm{cm} \mathrm{H}_{2} \mathrm{O}$ during maximal smooth muscle stimulation.

Figure 4 describes the relationships between changes in tracheal active tension and tracheal compliance. Because of technical factors, Bethanecol dosages of 0.04 and $0.08 \mathrm{mg} / \mathrm{kg}$ in

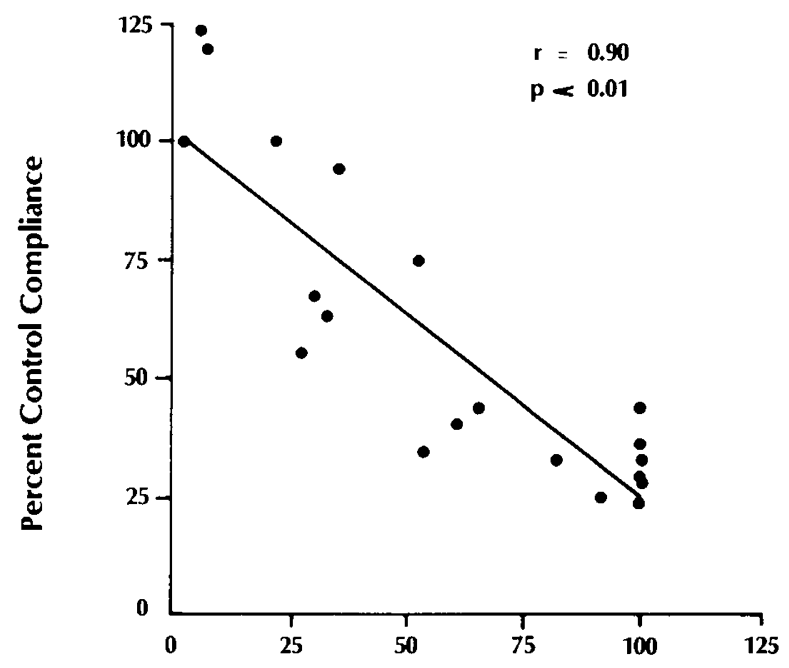

Percent Maximum Active Tension

Fig. 4. Percent control compliance is plotted against percent maximum active tension for individual lambs. (Data of lambs I and IV excluded, as explained in the text.)

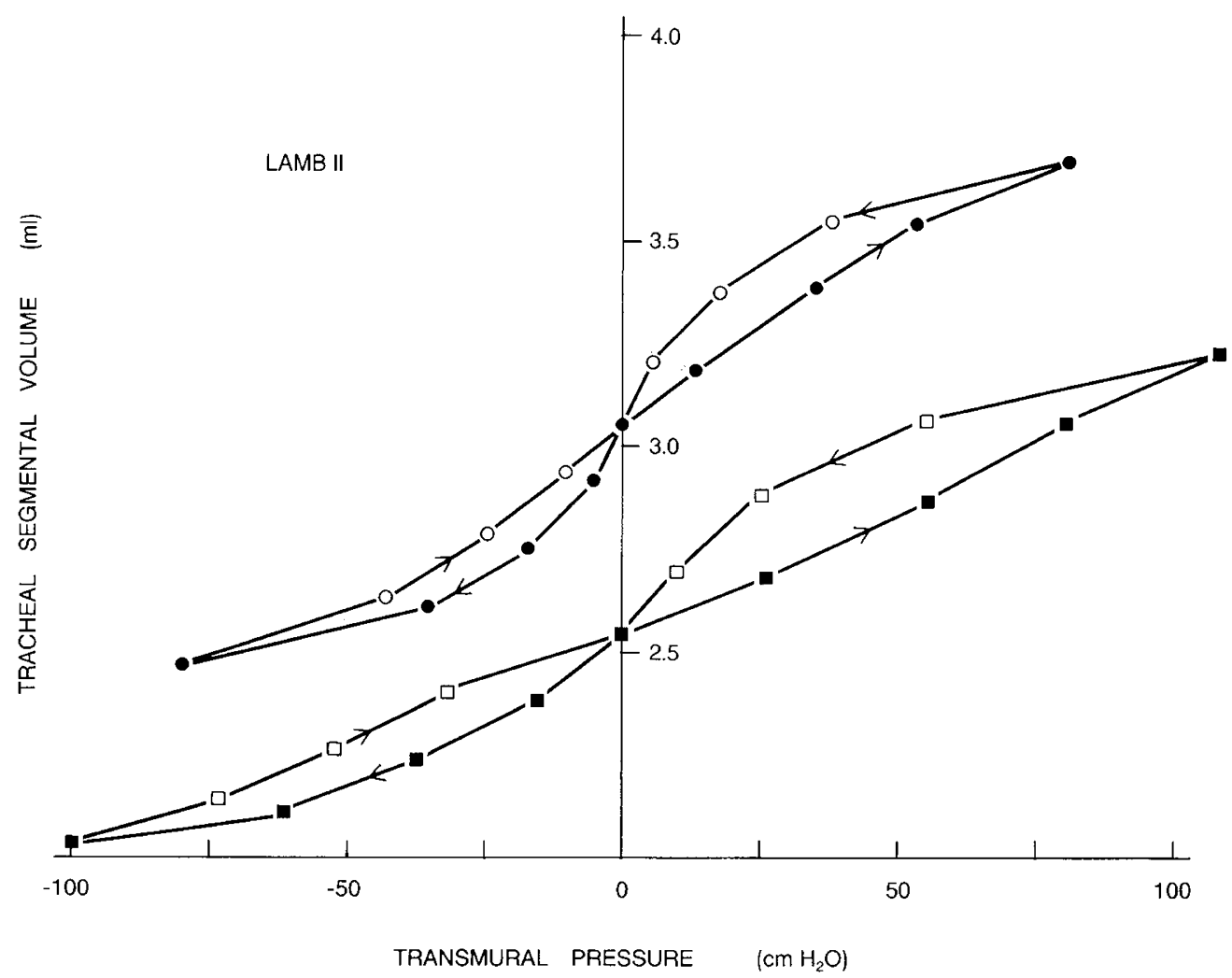

Fig. 3. Actual pressure-volume relationship (lamb no. II). Circles designate control conditions and squares indicate the experimental condition (maximal contraction). Closed points represent pressure changes

away from zero transmural pressure and open points indicate pressure changes back toward zero transmural pressure. 
lambs 1 and 4 demonstrated insignificant tension responses and were omitted from these data. Figure 4 illustrates that as maximum active tensions were approached, tracheal segments exhibited a decrease in compliance. These data demonstrate a significant linear relationship between developed active tension and tracheal compliance $(r=0.90, p<0.01)$.

As shown in Figure $5 \mathrm{~A}$ with maximum smooth muscle stimulation the relaxation time constant of the smooth muscle $\left(\tau_{\mathrm{R}}\right)$ was found to decrease by $18.4 \%(p<0.02)$ from the control level (mean values, $3.2 \pm 0.07 \mathrm{SEM} \mathrm{s).} \mathrm{In} \mathrm{addition,} \mathrm{the} \mathrm{tracheal}$ hysteresis was found to increase with increased Bethanecol level (Fig. $5 B)$. The mean control values $(5.01 \pm 0.69 \mathrm{SEM} \mathrm{g} \cdot \mathrm{cm})$ increased $82 \%(p<0.01)$ with maximum cholinergic stimulation.

\section{DISCUSSION}

The present study defines a spontaneously breathing newborn lamb model with an intact, bypassed tracheal segment that can

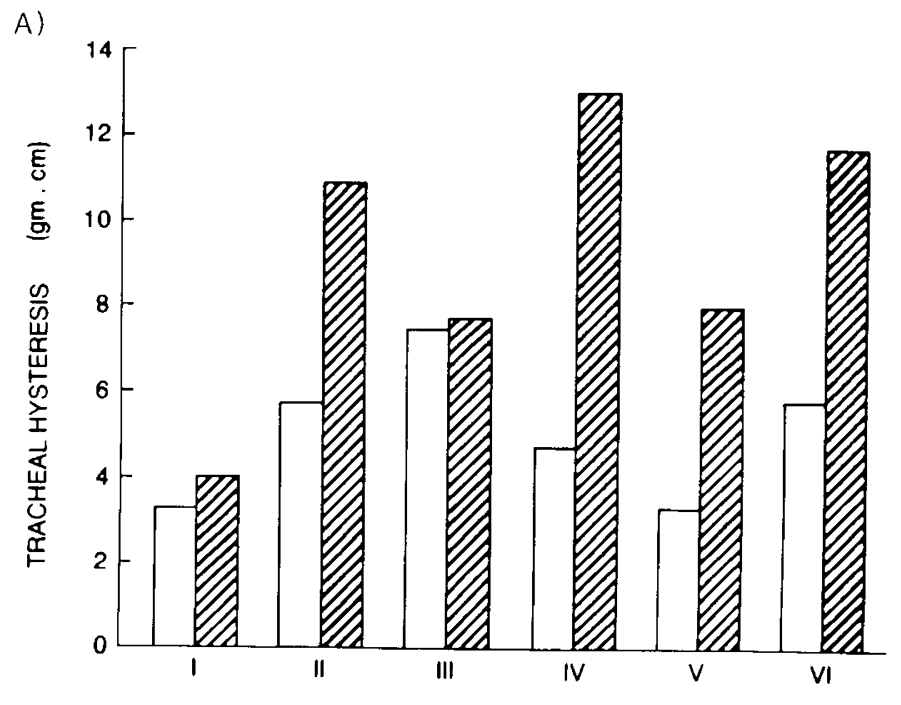

LAMB IDENTIFICATION NUMBER

B )

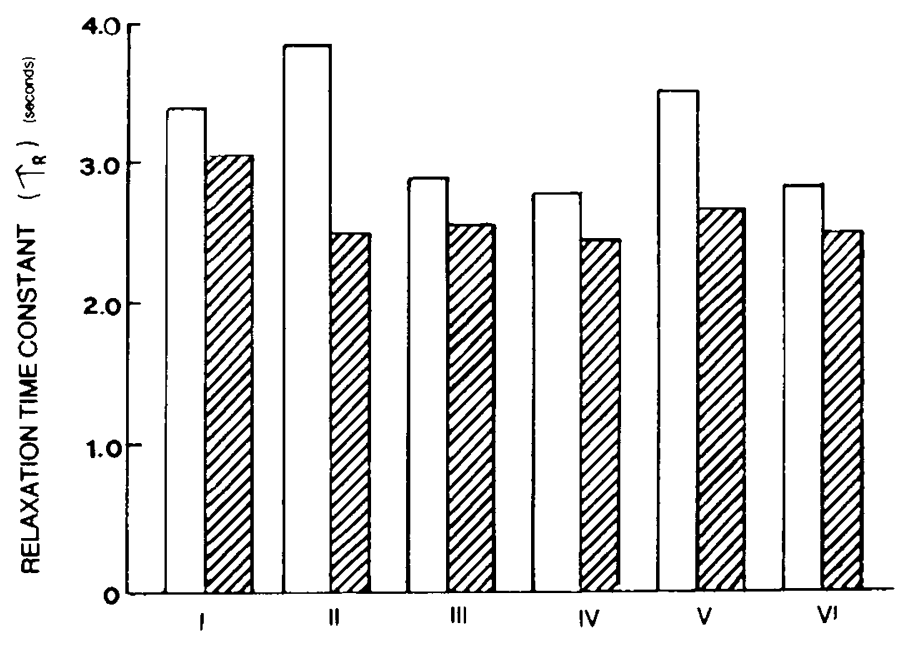

LAMB IDENTIFICATION NUMBER

Fig. 5. $A$, values of relaxation time constant $\left(\tau_{\mathrm{R}} \mathrm{S}\right)$ of the smooth muscle for control state and during maximal tracheal smooth muscle contraction for individual lambs; open bars represent control data and shaded hars represent experimental data $(0.16 \mathrm{mg} / \mathrm{kg}$ Bethanecol). $B$, tracheal hysteresis $(\mathrm{g} \cdot \mathrm{cm})$ comparing control (open bars) and experimental conditions (shaded bars, at $0.16 \mathrm{mg} / \mathrm{kg}$ of Bethanecol). be utilized to evaluate in vivo tracheal mechanics. The changes in the elastic and viscoelastic properties of the trachea were determined after incremental cholinergic stimulation. The data demonstrate that graded increases in the developed active tension were associated with decreased tracheal compliance and relaxation time constant of the smooth muscle and with an increase in tracheal hysteresis.

The sustained contractile response of the smooth muscle to a cholinergic agonist was documented. The tracheal segmental volume decreased significantly $(11.6 \%)$ with maximal stimulation. Although the integrity of the cholinergic receptors are indicated by these observations, this study does not address whether or not the parasympathetic pathway to the smooth muscle or receptors themselves are functional in the newborn. The fact that tracheal smooth muscle stimulation resulted in dimensional changes is of particular physiologic importance with respect to the neonatal anatomical dead space and airway resistance.

The role of airway smooth muscle differs from its effect on trachea to those on distal airways such as the alveolar duct. Tracheal smooth muscle is known to resist distension (9). At negative transmural pressures (as in forced expiration), invagination of the pars membrana is resisted and the tracheal smooth muscle tone opposes collapse of the adult airway (10). On the other hand, bronchial smooth muscle contraction results in bronchial narrowing but effective prevention of collapse (10). The ability of smooth muscle tone to stabilize distal airways is also expected but not confirmed (9). Therefore, the airway smooth muscle tone modulates two opposing characteristics of increasing airway resistance and reducing dead space ventilation. The changes induced by airway smooth muscle allows an individual to adapt to different patterns of ventilation. The ability of the neonatal tracheal smooth muscle tone to resist distension as in positive pressure ventilation, and oppose collapse, as during tracheomalacia-like changes secondary to airway barotrauma (11), needs to be investigated further.

Contraction of airway smooth muscle is also well known to alter the compressibility of the adult airway (8) by altering the tracheal mechanics (2). A significant correlation between neonatal lamb tracheal compliance and the incremental changes in the developed active tension have been demonstrated in our study. These data confirm that the neonatal tracheal preparation can exhibit rigidity when the trachealis tone is increased. Thus, the effect of compressive or collapsing transmural pressures on the compliant airway may be resisted by cholinergic control of airway rigidity. These changes in tracheal mechanics could be related to the contractile elements of the smooth muscle, to the effect of contraction on the passive components of the system, and to the activation state of the actin-myosin complex $(12,13)$.

Several experimental factors were considered while calculating the active tension developed upon cholinergic stimulation. Because the tracheal segment is not truly cylindrical, the application of the LaPlace relationship was limited to the inflation and deflation curve on the positive pressure limb of the P-V relationship. In addition, we determined the change in the developed active tension at isovolumic conditions and correlated the individual data to the respective tracheal compliance. Whether the fact that the trachea is a fibrocartilaginous structure in series with muscle has any effect on data analysis of LaPlace's equation is not known. Changes in tracheal volume and smooth muscle tension helped to confirm the vascular integrity of the tracheal model.

The viscoelastic properties evaluated in this study were the relaxation time constants of the tracheal smooth muscle and the tracheal hysteresis. The relaxation time constant characterizes the dissipation of the peak pressure to some steady state pressure following a saline step volume injection. Relaxation of these tissues permits accomodation of increased intraluminal volume with little change in pressure. Three phases of smooth muscle relaxation have been described: an early rapid phase attributed 
to collagen, a long slow phase attributed to smooth muscle, and finally the steady phase attributed to elastin (14). Both the adult tracheal $(2)$ and vascular $(3,14)$ stress relaxation time constant (estimated at about $2 \mathrm{HZ}$ ) have been observed to decrease upon smooth muscle stimulation. The rapidity with which the smooth muscle tone changes depends on its viscoelastic properties. The airway smooth muscle is slow relative to striated muscle and to the breathing frequencies. Its function appears to be to assume a state of contraction while dynamic breathing events continue, rather than participate actively in the breathing event itself (9). The relaxation time constant of the tracheal smooth muscle of the neonatal lamb was determined to be $3.2 \pm 0.07 \mathrm{~s}$ and also decreased significantly upon cholinergic stimulation. These values are higher than the stress relaxation time constant of vascular and adult airway smooth muscle.

The clinical significance of these data is that in the presence of maximal active tension, any change in tracheal volume consequent to a change in the transmural pressure will be retarded when the relaxation time constant is shortened. Depending on the compliance of the airways, considerable variations in tracheal-bronchial volume and hence its conductance and dead space would occur during a respiratory cycle (both spontaneous and mechanical ventilation). Thus, if there is a mechanism for the control of smooth muscle tone, these variations could be minimized.

High transmural pressures (as in positive pressure ventilation) and long durations of the relaxation time constants of the smooth muscle would mandate very slow rates of respiratory cycles so that a pressure change could be dissipated to a steady state. Because positive pressures are frequently applied at rates of 30 60 cycles/min, the relaxation time constant may play a significant role in tracheal wall stress and/or volume distension. Any shortening of the relaxation time constant may play an important role in reducing such deformation.

Tracheal hysteresis represents the energy lost from the airway during inflation and deflation; it is probably frictional and may be associated with tissue movement and interruption of the actinmyosin complex (15). The significant increase (82\%) in tracheal hysteresis may be only partially due to the viscous properties of the airway smooth muscle; the contribution of the vascular smooth muscle cannot be excluded.

Neonatal airway compliance is an important factor in airway vulnerability to barotrauma (11). Increased tracheal smooth muscle tone may provide a protective mechanism against pressureinduced deformation by increasing rigidity and tissue friction, by shortening the relaxation time constant, and by decreasing the magnitude of volume changes in a respiratory cycle. The tone of the trachealis muscle may also help to reduce dead space ventilation and resist both compressive and collapsing transmural pressures.

In conclusion, this study describes the effects of exogenous cholinergic stimulation on the elastic and viscoelastic properties of the neonatal airways. Presently, the effects of endogenous changes in smooth muscle tone, reflexly or by compensatory mechanisms, on both term and preterm neonatal airways are not known and need to be evaluated.

Acknowledgments. The authors thank Ms Shalini Dev for her editorial assistance and Ms Phyllis Sparrow for her secretarial assistance.

\section{REFERENCES}

1. Widdicombe JG 1963 Regulation of tracheobronchial smooth muscle. Physiol Rev 43:1-30

2. Coburn RF, Palombini B 1972 Time-dependent pressure-volume relationships of the in vivo canine trachea. Respir Physiol 16:282-289

3. Bobbaers H, Clement J, van Woestijne KP 1978 Dynamic viscoelastic properties of the canine trachea. J Appl Physiol 44:137-143

4. Mortola JP, Sant'Ambrogio G 1979 Mechanics of the trachea and behavior of its slowly adapting stretch receptors. J Appl Physiol 286:577-590

5. Kannan MS, Daniel EE 1980 Structural and functional study of control of canine tracheal smooth muscle. Am J Physiol 238:C27-33

6. Croteau JR, Cook CD 1961 Volume-pressure and length-tension measurements in human tracheal and bronchial segments. J Appl Physiol 16:170172

7. Bhutani VK, Rubenstein SD, Shaffer TH 1981 Pressure-volume relationships of trachea in fetal, newborn and adult rabbits. Respir Physiol 43:221-231

8. Palombini B, Coburn RF 1972 Control of the compressibility of the canine trachea. Respir Physiol 15:365-383

9. Stephens NL, Hoppin FG Jr 1986 Mechanical properties of airway smooth muscle. In: Handbook of Physiology, section 3: The Respiratory System; (section ed) Fishman A P; volume III, Mechanics of Breathing; Macklem PT, Mead J (volume ed). American Physiological Society, chapter 17, pp 263-276

10. Olsen CR, Stevens AE, Pride NB, Staub NC 1967 Structural basis for decreased compressibility of constricted tracheal and bronchi. J Appl Physiol 23:3539

11. Bhutani VK, Rubenstein D, Shaffer TH 1981 Pressure-induced deformation in immature airways. Pediatr Res 15:829-832

12. Hanks BSR, Stephens NL 1981 Mechanics and energetics of lengthening of active airway smooth muscle. Am J Physiol 241:C42-46

13. Gunst SJ, Russell JA 1982 Contractile force of canine tracheal smooth muscle during continuous stretch. J Appl Physiol 52:655-663

14. Dobrin PB 1978 Mechanical properties of arteries. Physiol Rev 58:397-460

15. Warshaw DM, Fay FS 1983 Cross-bridge elasticity in single smooth muscle cells. J General Physiol 82:157-199 